\title{
Psicooncología
}

ISSN: $1696-7240$

\section{Afrontamiento y bienestar psicológico en padres de niños y adolescen- tes con cáncer durante el tratamiento}

\author{
Cristina Velasco ${ }^{1, *}$; Camino Bengoechea²; Blanca López-Ibor ${ }^{3}$
}

Recibido: 16 de junio de 2019 / Aceptado: 5 de septiembre de 2019

Resumen: Objetivo: explorar la vivencia emocional de los padres de niños y adolescentes con cáncer de un modo más abierto y no exclusivamente centrado en búsqueda de consecuencias negativas para la familia. Método: Se realizó un estudio mixto (cuantitativo y cualitativo) a través de la realización de una entrevista semiestructurada a 30 padres de niños y adolescentes con cáncer en tratamiento en la Unidad de Hematología y Oncología Pediátrica de HM Montepríncipe. Resultados: Se han obtenido 5 temas principales de las entrevistas realizadas a los padres. Los padres de niños con cáncer durante el tratamiento de la enfermedad de su hijo emplean un afrontamiento activo, centrado en el presente que permite la aceptación. Por otro lado, los padres reflejan los modos de expresión emocional, la presencia de valores y crecimiento personal, la actitud de gratitud y el modelo de ayuda que han recibido en el lugar dónde ha sido atendido su hijo. En el modelo teórico se presenta la importancia y consistencia como predictor del bienestar psicológico (crecimiento personal y propósito vital) y la gratitud en esta población. Discusión/conclusiones: En los padres de niños y adolescentes con cáncer se debe favorecer un afrontamiento activo, permitiendo la importancia y consistencia con los valores y generando actitudes de gratitud y confianza que permitirán el crecimiento personal y el propósito vital de las familias, y por lo tanto una adecuada vivencia emocional en los padres resultará un beneficio directo para los hijos.

Palabras clave: Oncología pediátrica; cáncer infantil; adolescentes; familia: padres; afrontamiento; bienestar psicológico; gratitud; consistencia con los valores.

\section{[en] Parental coping and psychological well-being in pediatric oncolgy patients while on treatment}

\footnotetext{
Abstract: Our objective in this work has been exploring the parents emotional experiences while their children or adolescents with cancer were on treatment. We aproached them openly instead of exclusively focused on the known negative consequences of pediatric cancer in the family. Method: The population study included 30 parents whose children were on treatment at the Pediatric Hematology and Oncology Unit at Universitary HM Monteprincipe hospital. A mixed method study was carried out (quantitative and qualitative) through a semi-structured interview. Results: Five main findings have

1 Cristina Velasco. Departamento de Psicología y Pedagogía, Facultad de Medicina, Universidad San PabloCEU; Unidad de Hematología y Oncología Pediátrica HM Monteprincipe.

E-mail: cristina.velascovega@ceu.es

2 Camino Bengoechea. Unidad de Hematología y Oncología Pediátrica HM Monteprincipe. E-mail: cbengoechea@hmhospitales.com

3 López-Ibor, B. Unidad de Hematología y Oncología Pediátrica HM Monteprincipe E-mail: blopezibor@hmhospitales.com

* Dirección de correspondencia: Cristina Velasco. Departamento de Psicología y Pedagogía, Facultad de Medicina, Universidad San Pablo-CEU, Campus de Montepríncipe, 28925 Alcorcón, Madrid, Spain.

E-mail: cristina.velascovega@ceu.es
} 
been obtained from the parent's interviews. Parents of children with cancer use active coping, focused on the present therefore allowing acceptance during treatment of their child's illness. On the other hand, parents found an enviroment in the Unit in which they could express their emotions, their personal values, and their spiritual grothw. Gratitude towards life and caring was also and important finding in this study. In this theoretical model the comitment with values was a predictor of psychological wellbeing (personal growth and life sense) as well as gratitude. Discussion / conclusions: Active coping in parents of children and adolescents with cancer, should be encouraged in any Pediatric Oncology Unit, regarding commitment with values and attitudes of gratitude and trust. Gratitude allows personal growth and vital sense within families. An adequate emotional experience in parents results in a direct benefit on their children.

Keywords: Pediatric oncology; children's cáncer, adolecents, parents; coping; psychological wellbeing; gratitude; values commitment.

Sumario. 1. Introducción. 1.1. Afrontamiento en los padres de niños con cáncer 1.2. Bienestar en los padres de niños con cáncer 1.3. Gratitud y valores en los padres de niños con cáncer 2. Método 2.1. Descripción de la población de estudio 2.2. Procedimiento 2.3. Variables e instrumentos 3. Análisis de los datos. 4. Resultados 4.1. Afrontamiento activo, centrado en el presente y aceptación 4.2. Expresión emocional 4.3. Valores y crecimiento personal 4.4. Actitud de gratitud 4.5. Modelo de ayuda 4.6. Modelo teórico de bienestar, gratitud y valores en los padres de niños con cáncer 5. Discusión 6 . Referencias bibliográficas

Cómo citar: Velasco C, Bengoechea C, López-Ibor B. Afrontamiento y bienestar psicológico en padres de niños y adolescentes con cáncer durante el tratamiento. Psiconcología 2019; 16: 227-249. doi: $10.5209 /$ psic. 65589

\section{Introducción}

El cáncer infantil es una enfermedad grave, poco frecuente, que afecta a la vida del niño y a la de su familia. En España, se diagnostican al año aproximadamente 1.400 nuevos casos de cáncer en niños y adolescentes y dentro de los tipos de cáncer el más frecuente es la Leucemia, seguida de los tumores que afectan al Sistema Nervioso Central y los Linfomas ${ }^{(1)}$. En la actualidad, en Europa cerca del 80\% de los niños se curan y llegan a la vida adulta ${ }^{(2)}$. El cáncer infantil, que requiere un tratamiento complejo y largo en la vida del niño, afecta de forma determinante a la estructura personal y social de la familia. Por ello es una enfermedad que requiere una atención especializada, integral e interdisciplinar al niño o adolescente y a su familia ${ }^{(3-5)}$. Las principales etapas de la enfermedad son: "el diagnóstico, inicio del tratamiento, etapa de mantenimiento, fin de tratamiento, supervivencia a largo plazo, recaída y muerte"'(6,p.77).

En la Unidad de Hematología y Oncología Pediátrica del Hospital Universitario Madrid Montepríncipe el objetivo principal es que el niño y adolescente curado llegue a ser un adulto sano desde el punto de vista físico, psíquico, social y espiritual ${ }^{(3)}$. Se trata, por tanto, de apostar por el niño y su futuro, integrando la enfermedad en la vida normal del niño y de su familia. El equipo de profesionales (médicos pediatras oncólogos, enfermeras especializadas, auxiliares, fisioterapeuta, técnicos sanitarios y administrativos, psicólogo, musicoterapeuta, voluntarios y profesores con un colegio dentro de la Unidad) debe trabajar poniendo al niño en el centro de su trabajo ${ }^{(6)}$.

Los padres son figuras fundamentales para los hijos durante de la enfermedad. A partir del diagnóstico, comienzan un camino que hasta ese momento era desconocido 
y necesitan reorganizar sus vidas y las de su familia. Cuando se pregunta a los padres sobre sus funciones afirman que son proporcionar cuidados, ofrecer apoyo emocional, gestionar la reorganización familiar, ser el principal apoyo del niño y asumir la responsabilidad del día a día. Todo ello se puede resumir en "estar ahí"(7,8,9,10).

Tradicionalmente la mayoría de los estudios sobre los padres de niños con cáncer se han centrado en las consecuencias negativas que la enfermedad determina ${ }^{(9)}$. Sin embargo, estudios recientes señalan que los padres de niños con cáncer no muestran mayores niveles de problemas psicológicos, que la población normal y que el paso del tiempo es un factor que les ayuda a mejorar ${ }^{(11-13)}$. Por lo tanto, nos hemos planteado la necesidad de explorar la vivencia emocional de los padres de un modo más abierto y no exclusivamente centrado en búsqueda de consecuencias negativas para la familia.

\subsection{Afrontamiento en los padres de niños con cáncer}

El afrontamiento en los padres de niños con cáncer ha sido una variable ampliamente estudiada. En España, Espada y Grau ${ }^{(14)}$ realizaron un estudio cualitativo en el que clasificaron las estrategias de afrontamiento de los padres de niños con cáncer en activas, que a su vez pueden ser dirigidas al problema o dirigidas a la emoción, y pasivas. El afrontamiento del problema con entereza, la regulación afectiva aparentando normalidad, la adecuación a la acción, la calma, la búsqueda de información, y de apoyo social, la reevaluación o reestructuración cognitiva y la distracción son estrategias de afrontamiento activas. Estrategias pasivas son la rumiación, la evitación/huida y el aislamiento social Los padres de niños con cáncer en general usaban más las estrategias activas y adaptativas. En la misma línea, Norberg et al. ${ }^{(15)}$ analizaron las estrategias de afrontamiento que empleaban los padres de niños con cáncer en comparación con padres de niños que no tenían enfermedades graves. Los resultados mostraron que los padres de niños con cáncer empleaban más estrategias activas centradas en el problema que se han asociado con menores niveles de malestar, y en menor medida estrategias de evitación o pasivas.

Algunas de las estrategias que los padres ponen en marcha para ellos mismos o para ayudar a sus hijos durante el tratamiento de la enfermedad, son, por ejemplo, enseñar a sus hijos estrategias de cambio cognitivas, favorecer la expresión emocional, dar apoyo, facilitar información y buscar comprensión junto a estrategias de tipo práctico como dar la medicina o tomar decisiones concretas del día a día ${ }^{(16)}$. También la ayuda percibida, las creencias religiosas, el pensamiento positivo y la esperanza, el cuidado físico y psicológico de ellos mismos, proteger y mantener unida a la familia y vivir la vida durante y después del diagnóstico son estrategias funcionales que resultan de gran utilidad. Los padres además necesitan sentirse eficaces en el afrontamiento ${ }^{(17,18)}$. Por otro lado, la distracción es una de las estrategias evitativas más empleadas ${ }^{(16)}$.

\subsection{Bienestar en los padres de niños con cáncer}

El modelo del bienestar eudaimónico de Ryff( ${ }^{(19)}$ considera seis dimensiones: autoaceptación, relación positiva con los otros, autonomía, propósito vital, crecimiento personal y dominio del ambiente. Desde esta perspectiva, se pretende eliminar el reduccionismo propio de la perspectiva hedónica en el concepto del 
bienestar. Este modelo parece ser el más adecuado porque busca no sólo eliminar el malestar sino el desarrollo integral de la persona. El sentido vital y el propósito de vida aparecen como las dimensiones más consistentes con el bienestar eudaimónico porque se han relacionado con el afecto a corto y largo plazo ${ }^{(20)}$.

El propósito vital es aquella dimensión que hace referencia a los objetivos y metas que dan sentido a la vida de cada individuo ${ }^{(19)}$. Se puede decir que este concepto tiene relación con visiones existencialistas como la de Frankl ${ }^{(21)}$. En cada ser humano el significado y propósito de la existencia es único y específico. A la vez este sentido o propósito en la vida se relaciona con un sistema de valores que guía y estructura la propia vida.

Existe una carencia de estudios sobre el bienestar psicológico y sus variables asociadas en los padres y las familias de niños con cáncer $^{(9,22)}$, sin embargo, son fundamentales para entender la vivencia desde el enfoque eudaimónico. Entre las variables asociadas al bienestar subjetivo, la revisión realizada por Klassen et al. ${ }^{(23)}$ señala las relacionadas con la salud psicológica de los padres, y las características del niño (conducta y tiempo desde el diagnóstico) y los indicadores de afrontamiento (cohesión familiar, apoyo social, manejo del estrés). Por otro lado, Fotiadou et al. ${ }^{(24)}$ señaló que el optimismo en los padres se asociaba positivamente con la satisfacción con la vida, la percepción subjetiva de salud, así como el afrontamiento a través del apoyo social.

Anteriormente, Grootenhuis y Last ${ }^{(25)}$ habían señalado que el apoyo social juega un papel fundamental para el bienestar en las madres. Además, la comunicación abierta y frecuente sobre la enfermedad del niño y otros factores de protección como el optimismo, tener una visión positiva de la situación y mantener la esperanza se relacionaron con el bienestar en esta población. Recientemente, Toffalini et al. ${ }^{(26)}$ al estudiar el bienestar subjetivo en los padres de niños con cáncer afirmaron que la actitud que tienen y las evaluaciones que hacen ellos mismos sobre su vivencia influyen directamente en el bienestar. Por lo tanto, resulta relevante conocer no sólo cómo están viviendo la enfermedad de su hijo si no la evaluación que ellos mismos hacen de su vivencia.

\subsection{Gratitud y valores en los padres de niños con cáncer}

Son escasas las investigaciones dedicadas a estudiar la gratitud expresada por los padres de niños con cáncer, se puede destacar el estudio realizado por Laing y Moules ${ }^{(27)}$ en el que los padres tras asistir a un campamento con sus hijos expresan gratitud por el campamento en sí mismo, porque su hijo estuviese vivo y pudiese participar el él, así como por el descanso que había permitido en su vida diría y en su rutina. Esa gratitud fue presentada como fruto de ser verdaderamente conscientes del momento que estaban viviendo, de la finitud de la vida y de la fragilidad de la vida de su hijo. Además, en este mismo estudio, se señala la gratitud como un indicador de salud emocional permitiendo a los padres sentirse capaces de manejar toda la situación y vivencia de la enfermedad de su hijo. De igual manera en padres de niños con otras enfermedades, como padres de niños con artritis idiopática juvenil expresan esperanza y gratitud por la vida de su hijo al mismo tiempo que miedo y cansancio ${ }^{(28)}$.

Además, se ha encontrado relación entre la gratitud en las madres y la satisfacción vital de los hijos. De este modo, las madres con mayores índices de gratitud promueven en los hijos el sentido y el aprecio por las circunstancias propias de su vida ${ }^{(29)}$. 
Por otro lado, los valores son aquellos propósitos que encaminan a la acción y que la persona elige como guía para las direcciones que tienen un sentido o significado importante personal. En este sentido, la responsabilidad sobre las acciones, la persistencia y el cultivar las habilidades necesarias para llevarlos a cabo son elementos necesarios para el desarrollo de los valores. De este modo, la persona en primer lugar necesita clarificar sus valores para llevar a cabo una vida coherente con ellos, descubrir cuales son las direcciones valiosas para su vida. Una vez clarificados, la persona se implica en la realización de las acciones comprometidas con sus valores personales, que se traducen en las acciones que la persona lleva a cabo en su día a día ${ }^{(30)}$.

La literatura científica ha mostrado que los padres de niños con cáncer afirman experimentar cambios en las metas vitales y prioridades a la vez que expresan cambios positivos asociados a la experiencia incluyendo la reformulación de sus propios valores ${ }^{(31,32)}$. Ante la enfermedad grave de un hijo, los padres se encuentran con nuevas posibilidades. La disposición con la que viven estas posibilidades juega un papel fundamental. La esperanza ha sido considerada como un modo de dar sentido, y como resultado se realizan acciones coherentes con los propios valores $^{(31,33)}$. Por lo tanto, la consistencia con los valores y el cuidado del cultivo de estos, es uno de los ámbitos personales y familiares de relevancia para entender de un modo más completo la vivencia emocional de estas familias, en especial los padres que acompañan a su hijo a lo largo de todo el camino.

\section{Método}

Este estudio tiene como objetivo el desarrollo de un modo concreto de trabajar con el niño y con las familias y su influencia en la vivencia emocional de los padres. Como objetivo adicional, se pretende estudiar el afrontamiento de los padres, así como las variables psicológicas asociadas y aquello que resulta más adecuado para la ayuda integral al niño con cáncer y a su familia a través de un modelo de triangulación. Se pretende presentar un modelo de trabajo en oncología pediátrica desde la gratitud y la consistencia con los valores mediante el cual se pueda fomentar el bienestar en los padres de niños con cáncer.

\subsection{Descripción de la población de estudio}

La selección de la muestra se realizó a través de un muestreo intencional en la Unidad de Oncología y Hematología Pediátrica de HM hospitales/CIOCC situada en el hospital Montepríncipe. La población del estudio incluye un total de 25 familias a quiénes se invitó a participar a través de contacto telefónico. En dicho momento, uno de los investigadores (BLI) explicaba a los padres el objeto de la investigación y su plan de realización. Si estaban interesados en participar en ella, el investigador principal (CV) fue la responsable de llevar a cabo las entrevistas presenciales. Como criterio de inclusión en el estudio, el tiempo transcurrido desde el diagnóstico debía ser superior a un mes y el niño tenía que estar en tratamiento. Criterios de exclusión para participar en el estudio fueron la falta de contacto diario con el hijo y el fallecimiento del mismo. 
En el estudio participaron 20 familias, con un total 30 padres de niños que estaban actualmente recibiendo tratamiento en la Unidad de Oncología Pediátrica en el Hospital HM Montepríncipe. La media de la edad de los padres fue de $43,43(D T=6,02)$ con un rango de edad comprendido entre 28 y 52 . Todos los padres eran de nacionalidad española, la mayoría de los padres estaban casados y 4 de ellos divorciados. Los padres, en general, tenían un nivel educativo de formación media y superior. El 30\% de los padres evaluados había reducido su jornada laboral por enfermedad grave de un hijo mientras que el $40 \%$ continuaban trabajando a jornada completa. Más de la mitad de los padres habían variado su situación laboral por la enfermedad de su hijo. El 80\% de los padres tenían dos o más hijos. En la tabla 1. se presentan las características sociodemográficas de los padres.

Tabla 1. Características sociodemográficas de los padres

\begin{tabular}{|c|c|}
\hline $\mathbf{N}=\mathbf{3 0}$ & $\%$ \\
\hline \multicolumn{2}{|l|}{ Sexo } \\
\hline Hombre & 36,7 \\
\hline Mujer & 63,3 \\
\hline \multicolumn{2}{|l|}{ Estado civil } \\
\hline Casado & 86,7 \\
\hline Divorciado & 13,3 \\
\hline \multicolumn{2}{|l|}{ Nivel de Estudios } \\
\hline $\mathrm{ESO} / \mathrm{COU} / \mathrm{FP}$ & 30 \\
\hline Universitario & 70 \\
\hline \multicolumn{2}{|l|}{ Situación laboral } \\
\hline Tiempo completo & 40 \\
\hline Tiempo parcial & 13,3 \\
\hline Parado & 10 \\
\hline Ama de casa & 6,7 \\
\hline $\begin{array}{l}\text { Reducción de jornada por enferme- } \\
\text { dad grave de un hijo }\end{array}$ & 30 \\
\hline
\end{tabular}

En cuanto a los datos de los hijos, la media de edad de los hijos es de 8,90 $(\mathrm{DT}=4,71)$ comprendidos entre los 3 y los 17 años. La mayoría de los niños estaban en la fase de tratamiento activo (65\%). El diagnóstico más frecuente en los niños fue el de leucemia (30\%) seguido del de tumores del sistema nervioso central (SNC; 20\%). La media del tiempo desde el diagnóstico es de 10 meses y en todos los casos se trataba de un primer diagnóstico de cáncer. En la tabla 2. se presentan las características sociodemográficas y de salud de los hijos. 
Tabla 2. Características sociodemográficas y de salud de los hijos

\begin{tabular}{ll}
\hline $\mathbf{N}=\mathbf{2 0}$ & $\mathbf{\%}$ \\
\hline Sexo & 40 \\
$\quad$ Hombre & 60 \\
$\quad$ Mujer & \\
Enfermedad & 30 \\
$\quad$ Leucemia & 15 \\
Linfoma & 20 \\
Tumor SNC & 20 \\
Osteosarcoma & 10 \\
$\quad$ Sarcomas & 5 \\
Otros & \\
Fase de la enfermedad & \\
Tratamiento quimioterapia, radioterapia & 65 \\
y/o cirugía & \\
Mantenimiento & 25 \\
Rehabilitación secuelas & 5 \\
Post-trasplante & 5 \\
Tiempo desde el diagnóstico & \\
1 a 6 meses & 35 \\
7 a 12 meses & 25 \\
Más de 12 meses & 40 \\
\hline
\end{tabular}

\subsection{Procedimiento}

Los participantes respondieron a las preguntas a través de una entrevista semiestruturada y tres cuestionarios que evaluaban la gratitud, la importancia y consistencia con los valores y el bienestar (propósito vital y crecimiento personal). Las entrevistas con los padres fueron grabadas, trascritas y codificadas. En el método mixto de integración se recogen de modo simultáneo los datos de la investigación con lo que podremos validar los resultados obtenidos desde ambos enfoques. En el presente estudio lo que se busca es que ambos datos se confirmen mutuamente y resulten complementarios para la muestra de la atención de un modelo y poder conocer más.

\section{Teoría fundamentada}

Los componentes principales del método empleado por la Teoría Fundamentada ${ }^{(34)}$ son los siguientes: a) pregunta sobre un problema de investigación, b) recolección de datos y c) codificación. Así, la investigación cualitativa comienza con una pregunta problema abierta y flexible que se plantea el investigador acerca de una realidad o 
de un área del que se quiere ampliar los conocimientos existentes hasta el momento. Se trata de encontrar similitudes y diferencias de la información obtenida de los participantes del estudio para después interpretar los resultados. El método inductivo de análisis temáticos tiene su raíz en la Teoría Fundamentada y es uno de los métodos más empleados en los estudios mixtos sobre salud ${ }^{(35)}$.

Antes de iniciar cada entrevista, la evaluadora (CV) les entregó a los participantes el consentimiento informado y tras la obtención del consentimiento, comenzaba la entrevista, en la que se siguió, en todos los casos, el mismo orden de presentación de pruebas y preguntas. El estudio fue aprobado por el Comité de Ética HM hospitales.

Para la realización de la entrevista se obtuvo información del trabajo de campo realizado en la Unidad y se obtuvo la opinión de expertos en oncología pediátrica y en investigación sobre metodología cualitativa y mixta. Todos los expertos abordaron a través de reuniones aquellas preguntas que era adecuadas formular conforme a los objetivos del estudio. De este modo, los participantes fueron preguntados por su vivencia emocional y familiar, así como aquello que más les ayudaba en estos momentos. Un ejemplo de pregunta es: "En relación a los valores, personales y familiares, es decir aquello hacia lo que nos encaminamos en la vida, diría que han variado en el último mes", "Si le preguntase qué es lo mejor y lo peor de la situación que está viviendo, ¿qué me diría?" Algunas preguntas primarias fueron seguidas “¿Podrías decirme algo más?” Se trataba de generar un buen ambiente para la apertura y que hubiese dialogo. La media de la duración de las entrevistas fue de 30 minutos.

\subsection{Variables e instrumentos}

Los cuestionarios empleados han sido las Escalas de bienestar de Ryff ${ }^{(36)}$ con sus dos sub-escalas propósito vital y crecimiento personal, la escala de gratitud de McCulloungh et al. ${ }^{(37)}$ y la escala de importancia y consistencia de valores de Wilson y Luciano ${ }^{(38)}$.

a) Cuestionario de gratitud $(C G-6)^{(37,39)}$. El cuestionario evalúa la gratitud disposicional, es decir, la frecuencia e intensidad con la que la persona experimenta gratitud en su vida diaria. Se compone de un total de 6 ítems con formato de respuesta Likert $(1=$ totalmente desacuerdo, $7=$ totalmente de acuerdo).

b) Adaptación del Cuestionario de Valores propuesto por Wilson y Luciano ${ }^{(38)}$. Este cuestionario se compone de dos partes que evalúan 9 áreas de valor en dos partes. En la primera parte, evalúa la importancia de cada una de las áreas en una escala de $1 \mathrm{a} 10$, y, en una segunda parte se pregunta por la consistencia, es decir, cuánto ha estado trabajando la persona por cultivar esas mismas áreas. Las 9 áreas evaluadas han sido: Familia (diferente de esposo/a e hijos), esposo/a, pareja, relaciones íntimas, el cuidado de sus hijos, el cuidado de su hijo enfermo, amigos y vida social, trabajo, espiritualidad, crecimiento $\mathrm{y}$ aprendizaje (aprendiendo nuevas habilidades y adquiriendo conocimiento, o mejorándose como persona de la forma en que más le gustaría) y, por último, ocio, tiempo libre y descanso.

c) Escalas de propósito vital y crecimiento personal ${ }^{(36)}$. Estas escalas evalúan el bienestar psicológico a través de seis subescalas: propósito en la vida, 
dominio del entorno, relaciones positivas, autoaceptación, autonomía y crecimiento personal. El formato de respuesta es de tipo Likert $(0=$ totalmente en desacuerdo, 5 = totalmente de acuerdo). En el presente estudio se han empleado dos subescalas: el crecimiento personal y el propósito vital por ser aquellas que más se relacionan con el bienestar eudaimónico.

\section{Análisis de los datos}

El procedimiento de análisis de los datos obtenidos en las entrevistas se realizó con la ayuda del programa ATLAS TI 6.2 (software para investigación cualitativa). En primer lugar, se realizó el tipo de análisis inductivo el que se identifican los diferentes temas que emergen del análisis de contenido del discurso de los padres. Para ello, en primer lugar, se transcribieron los audios de las entrevistas por cada participante. A continuación, se indican una serie de temas principales y de estos temas surgen subtemas del análisis del contenido de las narrativas de los padres. Se trata de codificar la información, proceso mediante el cual se agrupa la información obtenida en temas que concentran las ideas en los temas similares. En segundo lugar, se generó un modelo teórico de relación de variables que son relevantes para los padres de niños con cáncer a través del programa SPSS.24 y de AMOS con ecuaciones estructurales. La última parte del estudio consistió en analizar la convergencia entre los datos obtenidos a través de la metodología cualitativa con los resultados obtenidos a través de los instrumentos empleados y el modelo de ecuaciones estructurales expuesto.

\section{Resultados}

Los resultados del estudio se presentan en dos fases. En primer lugar, se exponen los diferentes temas junto con las narrativas que emergen del análisis del contenido recogido en las entrevistas de tipo cualitativo de los padres de niños con cáncer que están actualmente recibiendo tratamiento. En segundo lugar, se presenta el modelo de ecuaciones estructurales de las variables evaluadas cuantitativamente. Por último, se realiza una integración de los resultados procedentes de los análisis cualitativo y cuantitativo que se expone en la discusión.

\subsection{Afrontamiento activo, centrado en el presente y aceptación}

Los padres de niños con cáncer afirman vivir el momento presente intentado disfrutar cada momento. Los padres comienzan a afrontar la enfermedad en el momento del diagnóstico y cada familia va elaborando sus modos de afrontamiento a lo largo de las distintas etapas de la enfermedad. Este afrontamiento se percibe principalmente en la fase de tratamiento, puesto que los padres perciben que sus hijos necesitan a padres activos e implicados en el cuidado. Según los padres entrevistados este proceso implicaría la vivencia del "aquí y el ahora" y experimentar los detalles del momento presente. En este sentido, los planes de futuro se trasforman en el presente, las familias entrevistadas afirman que no piensan demasiado en el futuro, si no que están en lo que ahora es importante para su vida. 
"Esta enfermedad te enseña a disfrutar de cada momento y a disfrutar del día a día porque mañana no sabes qué va a pasar. Yo sabía que iba a hacer todo lo posible por cuidar a mi hija porque no sabía si había futuro..." (padre de adolescente de 16 años)

"Al principio lo ves como con otra perspectiva, el temor ese siempre lo vas a tener... pero te cambia la perspectiva de futuro, tu futuro es hoy" (Madre de una adolescente de 15 años).

Esta posibilidad de estar presentes en todo momento durante la enfermedad de su hijo se hace posible gracias a la reducción de jornada por enfermedad grave de un $h i j o^{4}$. En España esta ayuda está destinada a los progenitores que reducen su jornada de trabajo para el cuidado del menor afectado por cáncer u otra enfermedad grave. De este modo se permite que los padres puedan conciliar el cuidado de su hijo con su vida laboral sin tener que renunciar ni a una ni a otra. Poder continuar con el trabajo permite a los padres dar normalidad a una vida en la irrumpe una enfermedad grave. A través de la manifestación del vivir el "aquí y el ahora" aparece en ellos la posibilidad de sentido. Vivir en el momento presente es una estrategia que permite a las familias la aceptación de la situación y de sus propias experiencias internas, sin embargo, el miedo al futuro, aun experimentando el presente, es algo que manifiestan la mayoría de los padres entrevistados.

"El futuro ahora mismo me da pavor... disfruto más del día a día, de las cosas presentes. He dejado de pensar en cosas a largo plazo, en fines de semana, vacaciones, que queda mucho de aquí al sábado... Me da hasta miedo lo de trabajar, pensar en que vuelvo" (Madre de un niño de 9 años).

"Tienes que encontrar tu para qué, lo que te sirve lo encuentras tú" (Madre de un adolescente de 17 años).

Otra de las vías a las que recurren algunos padres entrevistados para tratar de explicar el modo de afrontamiento es a través del hijo, en las entrevistas realizadas se ha observado que los padres refieren que el bienestar de sus hijos es aquello que les ayuda también a poner en marcha estrategias de afrontamiento activo y la aceptación.

"Aprendes a vivir el presente y sobre todo estoy contenta porque P. (hijo) está feliz, aquí viene feliz. El niño viene aquí contento. Claro que disfruto de las cosas a veces más que antes. Te vuelves como más dura. Te das cuenta de que hay cosas más esenciales. Vamos a valorar más la vida. Vas a disfrutar de la casa, de estar..." (Madre de un niño de 8 años).

"Desde luego que esto es la faena de tu vida, pero hay que asumirla, ayudar a vivir con esto, pero no dejar de vivir en ningún momento porque solo se vive una vez. Es el dolor más grande que yo he sentido nunca" (Madre de una niña de 4 años).

4 Real Decreto 1148/2011, de 29 de julio, para la aplicación y desarrollo, en el sistema de la Seguridad Social, de la prestación económica por cuidado de menores afectados por cáncer u otra enfermedad grave 
La aceptación de las emociones negativas, entre ellas, el miedo, el dolor, la tristeza, el enfado también es uno de los hechos que narran estos padres. Experimentar estas emociones y exponerse a ellas ya sea consigo mismo o con los demás es parte de la aceptación. Sin embargo, esa aceptación de las experiencias internas no es tan explícita, de hecho, hay padres que expresan que se quedan con lo bueno intentando olvidar lo malo:

"El terror, el miedo hay que aprender a convivir con ello. A veces el dolor era muy grande y te acomodas en la nube negra según el grado de dolor" (Padre de una niña de 10 años)

"Decirlo es parte de la aceptación, entonces no es fácil, solo lo pueden decir cuando te sientes comprendida, entonces el sentirte entendida es muy emocional. Hasta que no lo dices en voz alta... me dolía tanto que no podía decirlo en voz alta, solo escribía" (Madre de un adolescente de 16 años).

Los padres reflejan en sus entrevistas un afrontamiento activo, centrado en el problema, focalizados en el cuidado de su hijo y viviendo el día a día. Se puede decir que estas estrategias resultan de gran utilidad a los padres en la etapa del tratamiento de la enfermedad de su hijo.

\subsection{Expresión emocional}

Los padres expresan emociones y afirman que es importante encontrar los momentos para esto de diversos modos. Uno de los modos de expresión emocional, en el caso de la tristeza es el llanto, aunque a veces a los padres les costaba encontrar el momento para ello y también podían encontrar modos alternativos como expresar a través de la escritura.

"Me cuesta mucho llorar. Si cuento las cosas me siento el doble de peor. Me está ayudando mucho escribir, intento escribir sólo lo positivo, lo que estoy aprendiendo" (Madre de una niña de 13 años).

Por otro lado, el miedo en sus diferentes formas aparece ante la enfermedad grave hijo, los padres entrevistados hacían referencia a esta emoción como la más presente y la más difícil de manejar en las distintas etapas. El miedo es aquello que sucede sobre todo en los momentos iniciales cuando hay poca información y conocimiento de la situación de su hijo.

"El miedo absoluto, el abismo pues eso que te lo digan en el pasillo, estas como en una nube. A los dos días tienes que decir: "la niña está ahí y te tienes que rehacer" De ahí... ¿qué haces ahí lamentándote? Lo bueno de estar muy abajo es que solo hay una salida hacía la luz. No puedes cavar más en el hoyo porque será más lejana la salida (metáfora)" (Padre de una niña de 10 años). 
"Lo peor es el miedo a ver que tu hijo corre un riesgo para su vida. Miedo de ver que su vida peligra de golpe. Te levantas con una vida y te acuestas con otra" (Madre de un niño de 8 años).

La activación emocional implica una serie de consecuencias psicofisiológicas en los padres que serían necesarias abordar. Algunos de los padres entrevistados referían dificultades para dormir o cansancio.

“Al principio sí que me costaba dormir y también que estoy más cansada. Más que intranquila tiene que ver con el miedo. Al principio yo decía esto no es real" (Madre de una niña de 13 años)

Los padres entrevistados manifiestan que para que los demás te puedan ayudar y acompañar uno mismo tiene que contar lo que le sucede. De hecho, el hecho mismo de realizar la entrevista de alguna manera es un modo de expresión emocional.

"Es lo más duro que me ha pasado en mi vida, pero hay que levantarse enseguida. Yo creo que como intentes comerte todo esto solo sin comunicarlo, sin traducirlo, hay que estar muy abiertos, si no lo cuentas te mueres. Una persona solo te puede dar un abrazo o ayudar si sabe que lo necesitas" (Padre de una adolescente de 16 años).

La expresión emocional es otro modo de afrontamiento que puede resultar de utilidad para estas familias, aprender cómo y a quién contar aquello que les sucede es una constante a lo largo de toda la enfermedad.

\subsection{Valores y crecimiento personal}

Los padres durante el tratamiento de la enfermedad de su hijo afirman con contundencia que el principal valor en ese momento es cuidar de su hijo. Este permitirse el tiempo y el espacio para el cuidado de su hijo alienta el sentido, su propósito vital, y a la vez pone de manifiesto la meta de la curación y a la vez acompañar, guiar, educar, cuidar de sus hijos.

"Cuando estás en todo el meollo de la enfermedad no tienes tiempo de hacer otras cosas, no puedes, yo solo me dedico a L. (hija)" (Madre de una niña de 7 años).

"Mi meta es que mi hija salga adelante, el resto ya vendrá, asimilar y ver... Con la mejoría de la situación vuelven a ser importantes las cosas de antes" (Madre de una niña de 3 años).

Los padres entrevistados refieren una serie de cambios su vida familiar, así como una mejoría en algunos aspectos de esta, entre ellos, las relaciones familiares se trasforman siendo más profundas y han vivido una unión familiar fuerte durante el tratamiento. 
"Lo mejor puedo decir que es la relación con mi hija ha mejorado y también con mi marido porque nos ha unido" (Madre de una adolescente de 13 años).

"La enfermedad también te hace unirte, te hace hacer una piña para solucionar un grandísimo problema. Te ayuda a relativizar todo en la vida y a valorar lo verdaderamente importante que es la vida, la salud" (Padre de una adolescente de 16 años).

En esta última afirmación se puede percibir que, además de la mejora en las relaciones familiares, hay un cambio en los valores, en la perspectiva de vida. Compartir el tiempo con su hijo o sentirse unido al resto de la familia es el valor que comienza a tomar la mayor relevancia.

"Lo mejor el tiempo que compartes con ella (con su hija). Aunque estés en una situación que no te gustaría, pero te hace pasar tiempo con ella. El venir aquí y aprovecharlo para compartir con ella. En la vida real no hay tanto tiempo como ahora. Al igual que aprendes mucho de ella" (Padre de una adolescente de 13 años).

Desde ese cambio o nuevo enfoque de valores, los padres entrevistados hacen numerosas referencias a vivir los pequeños detalles de la vida, es decir, disfrutar aquello que te ofrece el día a día en las cosas normales pero realizadas en familia.

"Lo que disfrutas de las cosas normales, de la vida normal. Poder llevar a tu hijo al colegio, valorar sobre todo tu propia vida (...) Recuerdo el tiempo que llevó una sonda pues luego valoras que puede comer. Mi consejo puede ser: "déjate ayudar", yo hago todo lo que puedo por ayudar a mi hijo" (Madre de una niña de 4 años)

"El amor que siento hacía él, yo soy muy creyente y eso me ha ayudado, no he perdido la fe y luego con esperanza de que todo pasa, nada es eterno y de todo se aprende" (Madre de un niño de 7 años).

Sin embargo, algunos de los padres entrevistados refieren que se produce una falta de consistencia con algunos valores, por ejemplo, esta madre expresa como no está cuidando su área social que para ella es valiosa. Esta falta de consistencia con los valores les lleva a sentirse peor a largo plazo a la vez que sus hijos no quieren que sus padres dejen de hacer cosas por ellos.

"Mi vida en este momento es una mierda. Siento que un tsunami ha arrasado con mi trabajo, con mi marido, con mi hijo... A los amigos los tengo abandonados, no sé por dónde empezar a contarles" (Madre de una adolescente de 17 años);

"Abandonas todo, intentas integrar todo, el resto te da igual. Tú te vuelcas en todo, no he perdido las amistades gracias a que han estado muy receptivas. Mi hija me decía: "no quiero que dejes de hacer cosas por mí" (Madre de una adolescente de 16 años). 
Por último, desde esta perspectiva nueva de valores, siendo consistente con aquello que creen que tienen que hacer cada día, cultivando lo que para ellos y sus hijos es importante los padres dan un sentido a esa situación y la integran en su vida, en su historia.

"Al vivir esto creo que se refuerzan ciertos valores, lo bueno de la vida llega a aparecer. Y creo que a nivel personal tienes que asumir que lo que hay es lo que hay, es decir, o sea, intentar adaptarte, así puedes llegar a ver las cosas de otro modo" (Madre de una niña 6 años).

"A mí no me entra en la cabeza que un niño pueda tener esto, al final te das cuenta de que es tu historia" (Madre de un niño de 5 años).

Incluso llegan a afirmar que este cambio de valores los lleva a percibir todo lo que implica la enfermedad de su hijo como un regalo. Es en este punto cuando ellos al descubrir el sentido aparece la gratitud que se expone en el siguiente apartado por ser otra categoría encontrada en las narrativas.

"Un cambio de perspectiva a valorar lo bueno que tienes, los ratos con tu hijo. Yo disfruto hablando con él, ahora valoro más el tiempo con él. Ese cambio de perspectiva para valorar todo lo bueno que tienes es un don de Dios. El planteamiento es ¿te han quitado algo o te lo han dado? Ver como un regalo lo que se nos ha dado" (Padre de un niño de 4 años).

\subsection{Actitud de gratitud}

A lo largo de las entrevistas realizadas a los padres ha aparecido una actitud que es de gran ayuda para estas familias, la gratitud. Los padres entrevistados refieren estar agradecidos en primer lugar por la vida de su hijo, y en segundo lugar por toda la ayuda recibida en esta etapa de su vida.

"Lo mejor que de un dolor brutal sientes un sentimiento de amor, agradecimiento hacía muchas personas..." (Madre de un niño de 8 años).

"Mi experiencia me hace pensar que qué difícil y que por qué tiene que pasar esto, aunque rápidamente me siento agradecida" (madre de una niña de 4 años).

Este agradecimiento se puede hacer extensible a circunstancias que facilitan la conciliación del cuidado del hijo enfermo con el aspecto laboral o el hecho de tener intimidad, entre otros. Esta gratitud expresada también hacía todas las personas del equipo interdisciplinar que atiende a su hijo y a las personas en general. Esta situación les ha permitido redescubrir los valores del ser humano y también ellos mismos querer ayudar (es el caso de los padres que participaron en el estudio).

"Agradecida de tener el permiso de trabajo, de tener mi habitación solo... necesitas privacidad" (Madre de un adolescente de 17 años). 
"La gente tan maravillosa, B (médico), C (musicoterapeuta), J (fisioterapeuta), resto de la gente que me han ayudado mucho... estoy muy agradecida" (Madre de un niño de 10 años).

"Lo mejor se puede decir que es que he visto la parte buena de muchísima gente, he visto capacidad de entrega" (Madre de una adolescente de 16 años).

Aunque menos frecuente, alguno de los padres entrevistados no expresaba esta actitud de gratitud. El modelo teórico propuesto explica la variable gratitud de qué modo se puede ver fortalecida.

"Yo creo que todo el mundo, siempre en la vida queremos más, a todos nos falta algo, siempre te falta algo" (Madre de una niña de 5 años).

\subsection{Modelo de ayuda}

El lugar en el que son atendidos sus hijos cobra especial relevancia cuando se indaga sobre el afrontamiento y la vivencia de estos padres. Los padres entrevistados refieren que el funcionamiento de la Unidad resulta de gran ayuda para toda la familia, porque se les permite estar dedicados al cuidado de su hijo y confiar en la atención integral que allí recibe.

"Una de las cosas que me está ayudando es esta unidad, el ver bien los engranajes, cómo está todo el mundo pendiente del paciente y de los familiares, lo que es la atención integral. Cuando ves esto, te dejas llevar porque son los que saben" (Madre de una adolescente de 14 años); Y otra madre lo afirma de la siguiente manera: "Cuando llegas te sientes como que no sabes porque dónde ir y ella (médico) sabe dónde tocarte, entonces de repente te enseña el camino y sabes cómo organizarte, qué pasos dar" (Madre de una niña de 6 años).

Sentir confianza en el equipo de la Unidad les permite continuar cuidado a su hijo que es lo que realmente desean estar a su lado. La confianza se fomenta en primer lugar con la información, los padres necesitan que los profesionales estén disponibles para la escucha en todo momento.

"Yo creo que dejar trabajar a los médicos y profesionales, centrarte en hacer para tu hijo la situación más llevadera posible y disfrutar del tiempo extra que vas a estar con tu familia, en mi caso mi mujer y mi hijo" (Padre de un niño de 8 años).

"Lo que me parece muy bien es el equipo médico, la información que te dan. Se lo dijeron todo desde el primer momento, nos han dicho esto" (Madre de una niña de 13 años).

"El equipo ha sido increíble, a mi B (medico) me dio mucha esperanza. Me dijo: "volverá a jugar al futbol" eso es un chorro de esperanza. Por otro lado, volver a la normalidad también es sano" (Madre de un niño de 7 años). 
Algo que caracteriza de modo especial a la Unidad es que está adaptada al niño y a sus necesidades, los horarios son flexibles, los padres pueden disponer de tiempo porque los voluntarios se encargan de los niños si es necesario, disponen de un colegio que está dentro de la unidad. Los padres manifiestan sentirse muy bien atendidos desde este enfoque.

"Lo mejor es que aquí me han tratado fenomenal todo el equipo: P. (voluntaria) hablando conmigo, lo feliz que he visto a mi hija a pesar de lo duro de la enfermedad. Ahora disfruto más de A (mi hija). Otra cosa muy buena de aquí es que he tenido flexibilidad a la hora de poner a mi hija el tratamiento, por ejemplo, poder recibirlo por la tarde (Madre de una niña de 10 años).

El colegio dentro de la Unidad es el mejor reflejo de este modelo de ayuda que permite que los niños puedan continuar estudiando, es decir, haciendo aquello que le corresponde a un niño de su edad, así como seguirse desarrollando con otros niños con los que comparte clases, actividades, recreos, excursiones, etc.

"Cuando B (jefe de la unidad) me habló el primer día del colegio, dije; "esta mujer qué dice, a mí no me importa el colegio" Y luego lo he valorado muchísimo Es una idea fundamental para la curación de un niño. El sentirse no enfermo, haciendo lo que tiene que hacer" (Madre de una niña de 4 años).

Para el desarrollo del niño es fundamental tener a sus padres y a sus hermanos o abuelos, tíos, amigos, en el caso de los adolescentes, cerca. En la Unidad se facilita y promueve el contacto y se proporciona los lugares y actividades para que ellos también puedan estar. Los padres entrevistados afirman que la integración de la familia les permitió reorganizar mucho mejor toda su vida y afrontar de mejor modo esta etapa del tratamiento de la enfermedad

"Al inicio lo que más te preocupa cómo encajar toda la vida familiar... yo tengo otros dos hijos, su hermano ha podido venir aquí. M (hijo) necesitaba a su hermano. Tienes que tomar decisiones muy rápidas y cuando llegas aquí los médicos te van pautando el camino, ahora por aquí, ahora esto... y eso ayuda" (Madre de un niño de 6 años)

"Es la maravilla de este sitio es que puedes integrar a la familia aquí (...) también es importante que hayan podido venir sus amigos porque ha estado muy acompañada y le han dejado pasar de una manera muy agradable" (Madre de una adolescente de 16 años).

El modo de trabajar de la Unidad permite a los padres poder dar sentido a la experiencia de tener un hijo con una enfermedad grave como es el cáncer. Todos los profesionales ponen al niño, adolescente y familia en el centro de su trabajo de tal modo que todo se realiza pensando en él, aunque eso a veces suponga un esfuerzo a nivel profesional, personal y actitudinal. 


\subsection{Modelo teórico de bienestar, gratitud y valores en los padres de niños con cáncer}

En el modelo definitivo se representan aquellas variables que han mostrado relaciones significativas entre ellas siguiendo las indicaciones propuestas por Joreskog ${ }^{(40)}$. Las correlaciones entre las distintas variables se presentan en la tabla 3. El modelo de ecuaciones estructurales que se muestra en la tabla 4 y en la figura 1 que se presentan a continuación. En el modelo se puede ver como la gratitud aparece como variable independiente y de predisposición a generar bienestar e importancia y consistencia con los valores.

Tabla 3. Correlaciones entre variables cuantitativas

\begin{tabular}{ccc}
\hline & $\begin{array}{c}\text { Importancia y consistencia } \\
\text { con los valores }\end{array}$ & Bienestar psicológico \\
\hline Bienestar psicológico &, $784^{* *}$ & \\
Gratitud &, $648^{* *}$ &, $539^{* *}$ \\
\hline
\end{tabular}

Nota: $* * \mathrm{p}<0,01 ; * \mathrm{p}<0,05$

Tabla 4. Coeficientes de regresión no estandarizados para las variables analizadas

\begin{tabular}{ccccccc}
\hline & & & Estimate & S.E. & C.R. & P \\
\hline Gratitud & $<--$ & Gratitud &, 156 &, 034 & 4,581 & $* * *$ \\
Bienestar psicológico & $<---$ & $\begin{array}{c}\text { Bienestar } \\
\text { psicológico }\end{array}$ &, 345 &, 051 & 6,810 & $* * *$ \\
\hline
\end{tabular}

$* * * \mathrm{p} \leq, 001 ; * * \mathrm{p} \leq, 01 ; * \mathrm{p} \leq, 05 ; \mathrm{S}=$ Coeficiente regresión;

$\mathrm{SE}=$ error estándar; $\mathrm{CR}=$ proporción crítica

Como se puede ver en la tabla 5, el modelo propuesto muestra índices de ajuste excelentes siguiendo los criterios de ajuste propuestos por Scheiber, Nora, Stage, Barlow y King ${ }^{(41)}$. Debido al tamaño pequeño de la muestra los datos obtenidos en la Chi-cuadrado se deben interpretar con prudencia tomando en consideración con satisfactorios resultados obtenidos en el GFI, CFI, TLI y RMSEA.

Tabla 5. Índices de ajuste del modelo estructural propuesto

\begin{tabular}{|c|c|c|c|c|c|c|}
\hline$\chi^{2}$ & $\chi^{2} / \mathrm{gl}$ & $\mathbf{P}$ & GFI & CFI & TLI & RMSEA \\
\hline, 123 & 1 &, 725 &, 99 & 1,00 & 1,06 & 0,00 \\
\hline
\end{tabular}


Figura 1. Puntuaciones no estandarizadas del Modelo final de ecuaciones estructurales
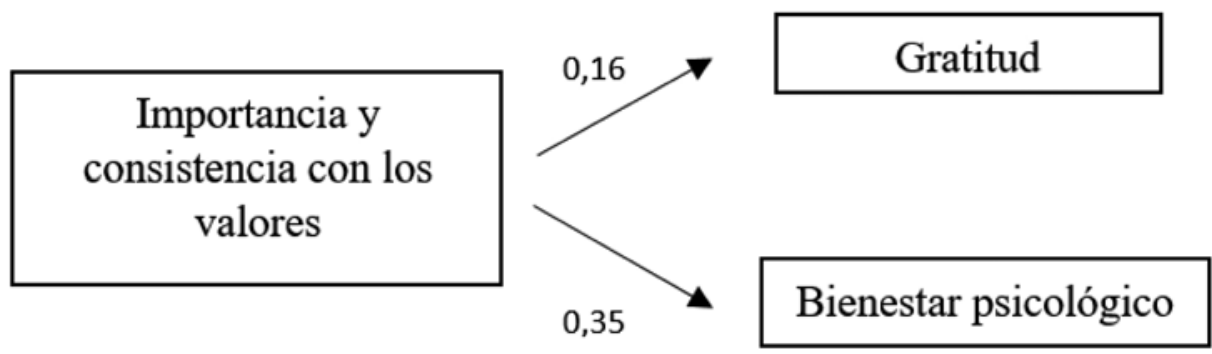

\section{Discusión}

Los resultados obtenidos del presente estudio tanto en las entrevistas como en los cuestionarios completados por los padres son muy significativos porque aportan información complementaria sobre el afrontamiento y la vivencia emocional de los padres de niños y adolescentes con cáncer. De este modo se pueden generar modelos de ayuda a estas familias. El modo de afrontamiento y el bienestar de los padres de niños con cáncer influyen de modo determinante en cómo el niño y adolescente se adapta a la enfermedad, los padres son las principales figuras de referencia en todas las etapas de la enfermedad. El niño aprende de sus padres también estrategias de regulación emocional ${ }^{(9,42)}$.

El modelo generado para explicar el bienestar psicológico de los padres pone de manifiesto la importancia y consistencia de los valores que la persona tiene, es una variable predisponente necesaria para experimentar un mayor crecimiento personal y un mejor propósito vital y gratitud ante la enfermedad de un hijo. Por otro lado, los modos de afrontamiento, entre ellos vivir el presente, los modos de expresión emocional, la expresión de gratitud y confianza a lo largo de todo el proceso y la reformulación de valores, así como el modelo de atención que han recibido permiten conocer cómo viven los padres de niños con cáncer la etapa del tratamiento.

De los resultados obtenidos en las entrevistas cabe destacar el modo de afrontamiento centrado en el presente, es decir, durante el tratamiento de la enfermedad de su hijo aquello que más les ayuda es vivir el día a día. Muchas veces está presente la incertidumbre, de hecho, los padres afirman que es la emoción que más experimentan a lo largo de toda la enfermedad. Se puede lidiar con la incertidumbre viviendo el momento presente, teniendo información y confiando en que todo el equipo está poniendo al niño y adolescente en el centro de su trabajo. En este sentido, dedicar tiempo a los padres en la fase del diagnóstico es fundamental. La jefa de la Unidad dedica el primer día de consulta con los padres y el niño o adolescente el tiempo para poder explicar cuál es la enfermedad, el tratamiento, así como responder a todas las cuestiones que puedan tener. En esta consulta la visión sobre el paciente va más allá de la enfermedad, se entiende a esa familia y a ese niño desde el plano físico, psicológico, social y espiritual. Mack y Grier ${ }^{(43)}$ hablan 
del periodo de incertidumbre con el que no normalmente llega una familia hasta que recibe el diagnóstico definitivo. Este diagnóstico debe ser hecho en un ambiente privado sin interrupciones y con realismo.

Estudios anteriores ya señalaban el beneficio que puede tener estar en contacto con el presente, tener capacidad para para percibir los recursos del presente y eso también ayuda a la aceptación y al sentido. Por lo tanto, el uso de estrategias activas y centradas en el presente coinciden con los resultados de estudios previos realizados con niños con cáncer ${ }^{15,17,18)}$. Sin embargo, evitar la expresión emocional puede tener consecuencias negativas para la salud psicológica de los padres ${ }^{(44)}$. Por lo tanto, fomentar el empleo de estrategias activas de afrontamiento va a beneficiar a estas familias.

Por otro lado, buscar y recibir ayuda de otras personas se incluye dentro de la estrategia de búsqueda de apoyo social, cuyos beneficios para los padres han sido señalados en estudios anteriores ${ }^{(45)}$. Además, los propios padres señalan que, si no compartes lo que te está sucediendo, y ahí estaría la expresión emocional, los demás no pueden ayudarte. Para que los demás puedan ayudarte es necesario compartir lo que te sucede, bien sea con familiares, amigos, profesionales, etc.

Un resultado novedoso del presente estudio es cómo los padres reflejan la confianza en el equipo como algo fundamental y puede estar relacionado con la actitud de gratitud y a su vez de aceptación. Para los padres tener información o bien tener la posibilidad de preguntar y de ser escuchados también promueve un mayor bienestar psicológico. Desde este modelo de ayuda se favorecería que los padres continúen realizando aquellos valores significativos para su vida personal y familiar.

Desde el modelo teórico se plantea la variable de importancia y consistencia con los valores como la variable que influye directamente en el propósito vital, en el crecimiento personal y en la gratitud expresada. Estos resultados se pueden unir a los obtenidos en las entrevistas. Los padres afirman que la organización de la Unidad les permitía llevar a cabo el cuidado de su hijo que es uno de los valores más importantes cuando irrumpe la enfermedad de un menor en una familia. Es decir, se trata de permitir a las familias cuidar de su hijo y de sus otros hijos, es decir, integrar la enfermedad en su vida normal ${ }^{(3)}$. Esta reformulación de valores, el experimentar cambios en sus metas vitales y dar lugar a esas nuevas posibilidades ha sido señalado como algo propio de los padres ante la enfermedad de un hijo ${ }^{(23,31)}$. Para dar sentido a toda esa vivencia es necesario que los padres puedan continuar realizando lo que es importante para ellos y para su familia.

En este sentido, los resultados de las variables dependientes muestran como en la medida en la que los padres hayan sido más consistentes con sus valores se hará más presente en ellos el propósito vital, el sentido de la vida y la gratitud. De igual manera la gratitud, expresada también en las entrevistas realizadas a los padres se muestra como un factor de protección que promueve el bienestar en los padres. Recientemente, hay estudios que señalan la relación entre la gratitud en los padres y en los hijos ${ }^{(29,46)}$. La gratitud es una fortaleza relacionada con el crecimiento personal ${ }^{(47)}$ que puede ayudar no solo en esta situación de la enfermedad si no a lo largo de toda su vida, fomentando un adecuado desarrollo psicológico y espiritual en el niño y adolescente con cáncer, este es uno de los objetivos presentes en la Unidad ${ }^{(3)}$. Cada familia aporta un significado único y específico de la experiencia, por lo tanto este propósito se relaciona con el sistema de valores que estructuran la propia vida, esta visión podría también fomentar en los padres el desarrollo de valores actitudinales ante situaciones de sufrimiento como es la enfermedad de un hijo ${ }^{(21)}$. 
Como implicaciones prácticas y de futuro es importante destacar que la extensión de los datos cualitativos se pretende generar modos de ayuda a estos padres que no aparecen recogidos en la información que se obtiene de los distintos autoinformes, de esta manera el estudio permite poder ampliar los modelos asistenciales integrales con las familias en oncología pediátrica. Además, el empleo de métodos mixtos de investigación ha demostrado ser de mayor utilidad cuando se trata de responder a preguntas complejas y sobre todo para la investigación en aquellas áreas de conocimiento en las que aún se conoce poco. El método mixto permite, por un lado, poder sistematizar la información y, por otro lado, no perder la riqueza contextual y toda la información situacional que se obtiene de los datos cualitativos. Este diseño de triangulación de los resultados se ha empleado por diferentes razones, entre otras la necesidad de presentar unidos o comparados resultados de diferentes tipos (cualitativos y cuantitativos) sobre variables poco estudiadas en oncología pediátrica y además pueden mostrar evidencia sobre un modo novedoso de entender la enfermedad pediátrica en la familia ${ }^{(48)}$. Para futuros estudios sería interesante evaluar a padres de niños y adolescentes en otras etapas del tratamiento, así como evaluar también variables asociadas en los hijos, para conocer de qué modo el bienestar de los padres condiciona el desarrollo de los hijos.

Como conclusion se puede decir que cuando los padres perciben que el equipo multidisciplinar de oncología pediátrica trabaja poniendo a su hijo en centro, son capaces de expresar, de pedir ayuda, de confiar y de sentir gratitud. Todas estas variables se han asociado a un mayor propósito vital y crecimiento personal en las familias. Este experimentar crecimiento personal y propósito vital durante el tratamiento de la enfermedad de un hijo permitirá actuar como factor de protección en las otras etapas de la enfermedad como puede ser el fin de tratamiento, la supervivencia a largo plazo o las recaídas y/o la muerte. Fomentar en las familias un afrontamiento activo, centrado en el presente, permitir la expresión emocional y la consistencia con los valores personales y familiares puede llevar a los padres a experimentar un crecimiento personal y gratitud que sirvan como escudo para continuar con su vida y la de su familia del modo más saludable posible.

\section{Referencias bibliográficas}

1. Peris R, Felipe S, Martínez N, Pardo E, Valero S. Cáncer infantil en España: Estadísticas 1980-2013. Valencia: Registro Nacional de Tumores Infantiles (RNTI-SEHOP); 2014.

2. Kaatsch P. Epidemiology of childhood cancer. Cancer Treat Rev 2010; 36:277-85. doi: 10.1016/j.ctrv.2010.02.003

3. López-Ibor B. Aspectos médicos, psicológicos y sociales del cáncer infantil. Psicooncología 2009; 6:281-84.

4. Méndez X, Orgilés M, López-Roig S, Espada JP. Atención psicológica en el cáncer infantil. Psicooncología 2004; 1:139-54.

5. Thaxter G, Stevens M, Craft M. et al. Childhood cancer - guidelines for standards of treatment \& care. Porto: ICCCPO Executive Committee adopted by SIOP; 2002.

6. Bengoechea C, López-Ibor B. Oncologia Pediatrica e musicoterapia. En: Cerlati P, Crivelli F., editores. Musicoterapia in oncologia e nelle cure palliative. Milano, Italia: FrancoAngely, 2015; p. 74-92. 
7. Ångström-Brännström C, Norberg A, Strandberg G, Söderberg A, Dahlqvist V. Parents' experiences of what comforts them when their child is suffering from cancer. J Pediatr Oncol Nurs 2010; 27:266-75. doi: 10.1177/1043454210364623

8. Clarke S, Davies H, Jenney M, Glaser A, Eiser C. Parental communication and children's behavior following diagnosis of childhood leukemia. Psychooncology 2005; 14:274-81. doi: $10.1002 /$ pon. 843

9. Eiser C. Children with Cancer: The Quality of Life. London: Lawrence Erlbaum Associates, Publishers, 2004.

10. Long KA, Marsland AL. Family adjustment to childhood cancer: A systematic review. Clin Child Fam Psychol Rev 2011; 14: 57-88. doi: 10.1007/s10567-010-0082-z

11. Dolgin MJ, Phipps S. Fairclough DL, Sahler OJZ, Askins M, Noll RB et al. Trajectories of Adjustment in Mothers of Children with Newly Diagnosed Cancer: A Natural History Investigation. J Pediatr Psychol 2007; 32: 771-82. doi: 10.1093/jpepsy/jsm013

12. Phipps S, Long A, Willard VW, Okado Y, Hudson M, Huang Q, et al. Parents of children with cancer: at-risk or resilient? J Pediatr Psychol 2015; 40: 914-25. doi: 10.1093/jpepsy/ jsv047.

13. Wijnberg-Williams BJ, Kamps WA, Klip EC, Hoekstra-Weebers JM. Psychological adjustment of parents of pediatric cancer patients revisited: five years later. Psychooncology. 2006; 15(1): 1-88. doi: 10.1002/pon.927

14. Espada MC, Grau C. Estrategias de afrontamiento en padres de niños con cancer. Psicooncología 2012; 9: 25-40.

15. Norberg AL, Lindblad F, Boman KK. Coping strategies in parents of children with cancer. Soc Sci Med 2005; 60: 965-75. doi: 10.1016/j.socscimed.2004.06.030

16. Hildenbrand AK, Alderfer MA, Deatrick JA. Marsac ML. A mixed methods assessment of coping with pediatric cancer. J Psychosoc Oncol 2014; 32: 37-58. doi:10.1080/073473 32.2013.855960

17. Fletcher P, Schneider M, Harry R. How do I cope? Factors affecting mothers' abilities to cope with pediatric cancer. J Pediatr Oncol Nurs 2010; 27: 285-98. doi: $0.1177 / 1043454209360839$

18. Miedema B, Hamilton R, Fortin P, Easley J, Matthews M. 'You can only take so much, and it took everything out of me': coping strategies used by parents of children with cancer. Palliat Support Care 2010; 8: 197-206. doi:10.1017/S1478951510000015

19. Ryff CD. Happiness is everything, or is it? Explorations on the meaning of psychological well-being. J Pers Soc Psychol 1989; 57: 1069-81. doi: 10.1037/0022-3514.57.6.1069.

20. Vázquez C, Hervás G, Rahona JJ, Gómez D. Psychological well-being and health: Contributions from Positive Psychology. Int J Clin Health Psychol 2009; 5: 15-28.

21. Frankl V. El hombre en busca de sentido. Barcelona: Herder, 1991.

22. Barlow JH, Ellard DR. The psychosocial well-being of children with chronic disease, their parents and siblings: an overview of the research evidence base. Child Care Health Dev 2006; 32: 19-31. doi: 10.1111/j.1365-2214.2006. 00591.x

23. Klassen A, Raina P, Reineking S, Dix D, Pritchard S, O’Donnell M. Developing a literature base to understand the caregiving experience of parents of children with cancer: a systematic review of factors related to parental health and well-being. Support Care Cancer 2007; 15: 807-18. doi: 10.1007/s00520-007-0243-X.

24. Fotiadou M, Barlow JH, Powell LA, Langton H. Optimism and psychological well-being among parents of children with cancer: an exploratory study. Psychooncology 2008; 17: 401-09. doi: 10.1002/pon.1257. 
25. Grootenhuis MA, Last B. Predictors of parental emotional adjustment to childhood cancer. Psychooncology1997; 6: 115-28. doi: 10.1002/1099-1611199706

26. Toffalini E, Veltri A, Cornoldi, C. Metacognitive aspects influence subjective well-being in parents of children with cancer. Psychooncology 2015; 24: 175-80. doi: 10.1002/ pon. 3622

27. Laing MC, Moules NJ. Stories from cancer camp: tales of glitter and gratitude. J Appl Hermeneutics 2014; 31: 1-16. doi: 10.1177/104345428700400116.

28. Gómez-Ramírez O, Gibbon M, Berard R, Jurencak R, Green J, Tucker L, et al. A recurring rollercoaster ride: a qualitative study of the emotional experiences of parents of children with juvenile idiopathic arthritis. Pediatr Rheumatol Online J 2016; 14:13. doi: 10.1186/ s12969-016-0073-9

29. Hoy B, Suldo S, Mendez L. Links between parents' and children's levels of gratitude, life satisfaction, and hope. J Happiness Stud 2013; 14: 1343-61. doi: 10.1007/s10902-0129386-7

30. Flaxman PE, Blackledge JT, Bond FW. Acceptance and commitment therapy: Distinctive features. New York: Routledge, 2010.

31. Hullmann SE, Fedele DA, Molzon ES, Mayes S, Mullins, LL. Posttraumatic growth and hope in parents of children with cancer. J Psychosoc Oncol 2014; 32: 696-707. doi: 10.1080/07347332.2014.955241

32. Klassen AF, Gulati S, Granek L, Rosenberg-Yunger ZRS, Watt L, Sung L, et al. Understanding the health impact of caregiving: a qualitative study of immigrant parents and single parents of children with cancer. Qual Life Res 2012; 21: 1595-605. doi: 10.1007/s11136-011-0072-8

33. Steger MF, Kashdan TB, Oishi S. Being good by doing good: Daily eudaimonic activity and well-being. J Res Pers 2009; 42: 22-42. doi:10.1016/j.jrp.2007.03.004.

34. Strauss AL, Corbin J, Zimmerman E. Bases de la investigación cualitativa: técnicas y procedimientos para desarrollar la teoría fundamentada. Medellin: Editorial Universidad de Antioquia, 2002.

35. Hildenbrand AK, Alderfer MA, Deatrick JA, Marsac ML. A mixed methods assessment of coping with pediatric cancer. J Psychosoc Oncol 2014; 32: 37-58. doi:10.1080/073473 32.2013.855960

36. Díaz D, Rodríguez-Carvajal R, Blanco A, Moreno-Jiménez B, Gallardo I, Valle C, van Dierendonck D. Adaptación española de las escalas de bienestar psicológico de Ryff. Psicothema 2006; 18: 572-77.

37. McCullough ME, Emmons RA, Tsang JA. The grateful disposition: A conceptual and empirical topography. J Pers Soc Psychol 2002; 82: 112-27. doi: 10.1037/00223514.82.1.112

38. Wilson KG, Luciano MC. Terapia de Aceptación y Compromiso (ACT). Madrid: Ediciones Pirámide, 2002.

39. Beléndez M. Traducción y adaptación al castellano del cuestionario de Gratitud disposicional (CG-6). 2011; [Documento pdf] Universidad de Alicante. [Acceso 10 septiembre de 2019]. Disponible en: http://www.psy.miami.edu/faculty/mmccullough/ GratitudeRelated\%20Stuff/Spanish_gratitude_scale_Belendez2011.pdf

40. Joreskog KG. Testing structural equation models. En Bollen KA, Lang JA. editors. Testing structural equation models. Newbury Park, CA: Sage, 1993. pp. 294-316.

41. Schreiber JB, Nora A, Stage FK, Barlow EA, King J. Reporting structural equation modeling and confirmatory factor analysis results: A review. J Educ Res 2006; 99: 32338. doi:10.3200/JOER.99.6.323-338 
42. Morris AS, Silk JS, Steinberg L, Myers SS, Robinson LR. The role of the family context in the development of emotion regulation. Soc Dev 2007; 16:361-88. doi: 10.1111/j.14679507.2007.00389.x

43. Mack J, Grier H. The day one talk. J Clin Oncol 2004; 22: 563-6. doi: 10.1200/ JCO.2004.04.078

44. Cernvall M, Carlbring P, Ljungman G, von Essen L. Guided self-help as intervention for traumatic stress in parents of children with cancer: conceptualization, intervention strategies, and a case study. J Psychosoc Oncol 2013; 31: 13-29. doi: 10.1080/07347332.2012.741095

45. Hoekstra-Weebers JM, Jaspers JPC, Kamps WA, Klip EC. Psychological adaptation and social support of parents of pediatric cancer patients: a prospective longitudinal study. J Pediatr Psychol 2001; 26: 225-35. doi: 10.1093/jpepsy/26.4.225

46. Rothenberg WA, Hussong AM, Langley HA, et al. Grateful parents raising grateful children: Niche selection and the socialization of child gratitude. Appl Dev Sci 2017; 21:106-20. doi:10.1080/10888691.2016.1175945.

47. Peterson C, Seligman MEP. Character strenghts and virtues. New York: Oxford University Press; 2004.

48. Creswell J, Plano V. Designing and conducting mixed methods research. Thousand Oaks, CA: Sage, 2007. 